\title{
PERIODIC MODULES WITH LARGE PERIODS
}

\author{
JON F. CARLSON ${ }^{1}$
}

\begin{abstract}
Let $G$ be a nonabelian group of order $p^{3}$ and exponent $p$, where $p$ is an odd prime. Let $K$ be a field of characteristic $p$. In this paper it is proved that there exist periodic $K G$-modules whose periods are $2 p$. Some examples of such modules are constructed.
\end{abstract}

1. Introduction. Let $G$ be a finite $p$-group, and let $K$ be a field of characteristic $p$, where $p$ is a prime integer. If $M$ is a $K G$-module then there exists a projective $K G$-module $F$ such that there is an epimorphism $\varphi$ : $F \rightarrow M$. The kernel of $\varphi$ can be written as $\Omega(M) \oplus E$ where $E$ is projective and $\Omega(M)$ has no projective submodules. It is well known [5] that the isomorphism class of $\Omega(M)$ is independent of the choice of $F$ and $\varphi$. Inductively we define $\Omega^{n}(M)=\Omega\left(\Omega^{n-1}(M)\right)$ for all integers $n>1$. A $K G$ module is said to be periodic if there exists an integer $n>0$ such that $M \simeq \Omega^{n}(M) \oplus P$ where $P$ is a projective $K G$-module. If $n$ is the smallest such integer then $n$ is called the period of $M$.

Recently it has been proved that, when $G$ is abelian, every periodic $K G$-module has period 1 or 2 (see [2] or [4]). It is well known that if $G$ is a quaternion group, then every $K G$-module has period 1,2 or 4 . Until now there were no known examples of periodic modules with periods other than 1 , 2 , or 4 (see [1]). In this paper we show that if $G$ is a group of order $p^{3}$ and exponent $p$ for $p$ an odd prime, then there exist periodic $K G$-modules with period $2 p$. Some examples along with their minimal projective resolutions are explicitly constructed.

2. Notation and preliminaries. Let $p$ be an odd prime integer. Suppose that $K$ is a field of characteristic $p$. Throughout the rest of this paper $G$ will denote the group of order $p^{3}$ and exponent $p$. Then $G$ is generated by two elements $x$ and $y$. If $z=x^{-1} y^{-1} x y$, then we have the relations $x^{p}=y^{p}=z^{p}=1$, $x z=z x$, and $y z=z y$. Let $H$ be the subgroup generated by $x$ and $z$. Let

$$
\tilde{H}=\sum_{h \in H} h=(x-1)^{p-1}(z-1)^{p-1} \in K H .
$$

Recall that a $K H$-module $L$ is free if and only if $\operatorname{Dim}_{K} \tilde{H} L=\left(1 / p^{2}\right) \operatorname{Dim}_{K} L$. If $M$ is a $K G$-module, then $M_{H}$ is its restriction to a $K H$-module.

Received by the editors September 28, 1978.

AMS (MOS) subject classifications (1970). Primary 20C05, 16A26, $20 \mathrm{C20.}$

Key words and phrases. Periodic modules, modular group algebras.

${ }^{1}$ This work was partially supported by NSF Grant No. MCS 78-01685.

(c) 1979 American Mathematical Society 0002-9939/79/0000-0404/\$02.75 
The remainder of this section is devoted to establishing some combinatorial relations which will be needed in the next section. For $\alpha \in K$, let $l=l(\alpha)=$ $(y-1)-\alpha(x-1) \in K G$.

LEMMA 2.1. $l^{p}=k(z-1)^{p-1}$ where

$$
k=\sum_{t=1}^{p-1}(-1)^{t} \frac{1}{p}\left(\begin{array}{c}
p \\
t
\end{array}\right) \alpha^{t} x^{t} y^{p-t} .
$$

Proof. Note that $l=(\alpha-1)+(y-\alpha x)$. Therefore

$$
l^{p}=(\alpha-1)^{p}+\left(y-\alpha x^{p}\right)=\alpha^{p}-1+(y-\alpha x)^{p} .
$$

In the expansion of $(y-\alpha x)^{p}$ the coefficient on $(-\alpha)^{t}$ is the sum of all possible products of $x$ 's and $y$ 's with $x$ occurring $t$ times and $y$ occurring $p-t$ times. Each such product can be written in the form $x^{t} y^{p-t} z^{s}$ for some $s=0,1, \ldots, p-1$. Suppose that in each product the left-most letter (either $x$ or $y$ ) is moved to the furthest right position. This operation amounts to multiplying the product by $z^{t}$. However the entire sum remains unchanged. Hence for $t=1, \ldots, p-1$, the coefficient on $(-\alpha)^{t}$ is

$$
\sum_{j=0}^{p-1} \frac{1}{p}\left(\begin{array}{c}
p \\
t
\end{array}\right) x^{t} y^{p-t} z^{j}=\frac{1}{p}\left(\begin{array}{c}
p \\
t
\end{array}\right) x y^{p-t}(z-1)^{p-1} .
$$

This proves the lemma.

LEMMA 2.2. There exists an element $\nu \in K G$ such that $l k-k l=\nu(z-1)$. Moreover if $\alpha$ is an element of $K$ which is not in the prime field $F_{p}$, then $\nu$ is a unit in $K G$.

Proof. Now $l k-k l=(y k-k y)-\alpha(x k-k x)$. So

$$
\begin{aligned}
l k-k l= & \sum_{t=1}^{p-1}(-1)^{t} \frac{1}{p}\left(\begin{array}{c}
p \\
t
\end{array}\right) \alpha^{t} x y^{p-t+1}\left(z^{-t}-1\right) \\
& -\sum_{t=1}^{p-1}(-1)^{t} \cdot \frac{1}{p}\left(\begin{array}{c}
p \\
t
\end{array}\right) \alpha^{t+1} x^{t+1} y^{p-t}\left(1-z^{t}\right) .
\end{aligned}
$$

Note that $z^{-t}-1=z^{p-t}-1=(z-1)\left(z^{p-t-1}+\cdots+z+1\right)$. Hence $l k$ $-k l=\nu(z-1)$ where

$$
\begin{aligned}
\nu= & \alpha x z^{-1}-\alpha^{p} y z^{-1} \\
& -\sum_{t=1}^{p-2}(-1)^{t} \frac{1}{p}\left[\left(\begin{array}{c}
p \\
t+1
\end{array}\right)\left(z^{p-t-2}+\cdots+1\right)\right. \\
& \left.\quad-\left(\begin{array}{c}
p \\
t
\end{array}\right)\left(z^{t-1}+\cdots+1\right)\right] \alpha^{t+1} x^{t+1} y^{p-t} .
\end{aligned}
$$

Let $\epsilon: K G \rightarrow K$ be the augmentation homomorphism given by $\epsilon(g)=1$ for all $g \in G$. Then 


$$
\begin{aligned}
\epsilon(\nu) & =\alpha-\alpha^{p}-\sum_{t=1}^{p-2}(-1)^{t}\left[\frac{1}{p}\left(\begin{array}{c}
p \\
t+1
\end{array}\right)(-t-1)-\frac{1}{p}\left(\begin{array}{c}
p \\
t
\end{array}\right)(t-p)\right] \alpha^{t+1} \\
& =\alpha-\alpha^{p} .
\end{aligned}
$$

The lemma follows from the fact that $\nu$ is a unit if and only if $\epsilon(\nu) \neq 0$.

Now if $r=1, \ldots, p-1$, then

$$
\begin{aligned}
l^{r} k-k l^{r} & =\sum_{j=0}^{r-1} l^{j}(l k-k l) l^{r-1-j} \\
& =\sum_{j=0}^{r-1} l^{j} \nu l^{r-1-j}(z-1) .
\end{aligned}
$$

Therefore

$$
\left(l^{r} k-k l^{r}\right)(z-1)^{p-2}=r l^{r-1} \nu(z-1)^{p-1} .
$$

3. The main result. Let $K$ be a field with odd characteristic $p$. Let $G$, $l=l(\alpha), k, \nu$ be as in the previous section. Let $W$ be the left ideal in $K G$ given as $W=K G l+K G(z-1)$. We define $M=M(\alpha)=K G / W$. Then $M$ is a cyclic $K G$-module generated by $m=1+W$ where $(z-1) m=0$ and $(y-1) m=\alpha(x-1) m$. The dimension of $M$ is $p$, and the restriction of $M$ to a $K\langle x\rangle$-module is isomorphic to $K\langle x\rangle$.

THEOREM 3.1. If $\alpha \notin F_{p}$ (the prime field), then $M(\alpha)$ is a periodic $K G$-module with period $2 p$.

The proof consists of constructing a minimal free resolution for $M$. Let $F=K G a \oplus K G b$ be a free module with generators $a$ and $b$. For each $i=1, \ldots, p-1$, let

$$
\begin{aligned}
& m(i, 1)=l^{i} a-(z-1) b, \\
& m(i, 2)=k(z-1)^{p-2} a-l^{p-i} b .
\end{aligned}
$$

Define $M_{i}$ to be the submodule of $F$ generated by $m(i, 1)$ and $m(i, 2)$.

LEMMA 3.2. $\operatorname{Dim} M_{i}>p^{3}+p$.

Proof. Now $\tilde{H} m(i, 1)=(y-1)^{i} \tilde{H} a$, and $\tilde{H} m(i, 2)=-(y-1)^{p-i} \tilde{H} b$. Therefore the $K H$-module

$$
E=\sum_{j=0}^{p-i-1} K H(y-1)^{j} m(i, 1) \oplus \sum_{j=0}^{i-1} K H(y-1)^{j} m(i, 2)
$$

is a free $K H$-submodule of $\left(M_{i}\right)_{H}$ whose $K H$-socle is the subspace with a basis consisting of the elements $(y-1)^{t} \tilde{H} a, t=i, \ldots, p-1$, and $(y-1)^{s} \tilde{H} b$, $s=p-i, \ldots, p-1$. Also $\operatorname{Dim} E=p^{3}$. Let

$$
\begin{aligned}
m(i, 3) & =k(z-1)^{p-2} m(i, 1)-l^{i} m(i, 2) \\
& =\left(k l^{i}-l^{i} k\right)(z-1)^{p-2} a \\
& =-i l^{i-1} \nu(z-1)^{p-1} a .
\end{aligned}
$$


The last equality follows from (2.3). By Lemma 2.2 ,

$$
(x-1)^{p-1} \nu^{-1} m(i, 3)=-i(y-1)^{i-1} \tilde{H} a \notin E .
$$

Let

$$
L=K H \nu^{-1} m(i, 3)=K\langle x\rangle \nu^{-1} m(i, 3) .
$$

Then $L \cap E=0$ and $\operatorname{Dim} L=p$. Consequently $L \oplus E$ is a subspace of $M_{i}$ of dimension $p^{3}+p$.

LEMMA 3.3. $M_{1} \simeq \Omega^{2}(M)$ and $\operatorname{Dim} M_{1}=p^{3}+p$.

Proof. From the definition we know that $W \simeq \Omega(M)$. We have an exact sequence

$$
0 \rightarrow \Omega^{2}(M) \rightarrow F \stackrel{\varphi}{\rightarrow} W \rightarrow 0
$$

where $\varphi$ is defined by $\varphi(a)=z-1$ and $\varphi(b)=l$. Then

$$
\varphi(m(1,1))=l(z-1)-(z-1) l=0 .
$$

Also $\varphi(m(1,2))=k(z-1)^{p-1}-l^{p}=0$, by Lemma 2.1 . Hence $M_{1}$ is in the kernel of $\varphi$. Since $\operatorname{Dim} W=p^{3}-p$, the dimension of the kernel of $\varphi$ is $p^{3}+p$. By Lemma 3.2, $M_{1}$ is the kernel of $\varphi$.

LEMMA 3.4. For each $i=1, \ldots, p-2, \Omega^{2}\left(M_{i}\right) \simeq M_{i+1}$. Moreover $\operatorname{Dim} M_{i}$ $=p^{3}+p$ for all $i=1, \ldots, p-1$.

Proof. Assume, by induction, that $\operatorname{Dim} M_{i}=p^{3}+p$. Note that

$$
l^{p-i} m(i, 1)-(z-1) m(i, 2)=0 \text {. }
$$

We also have that

$$
\begin{aligned}
l k(z-1)^{p-2} m(i, 1)-l^{i+1} m(i, 2) & =-l\left(l^{i} k-k l^{i}\right)(z-1)^{p-2} a \\
& =-i \nu(z-1)^{p-1} m(i, 1) .
\end{aligned}
$$

Therefore

$$
[l k+i \nu(z-1)](z-1)^{p-2} m(i, 1)-l^{i+1} m(i, 2)=0 .
$$

Let $F^{\prime}=K G c \oplus K G d$ be the free $K G$-module with generators $c$ and $d$. We can form the exact sequence

$$
0 \rightarrow \Omega\left(M_{i}\right) \rightarrow F^{\prime} \stackrel{\psi}{\rightarrow} M_{i} \rightarrow 0,
$$

where $\psi(c)=m(i, 1)$ and $\psi(d)=m(i, 2)$. By (3.5) and (3.6), the kernel of $\psi$ contains the elements

$$
u_{1}=l^{p-i} c-(z-1) d
$$

and

$$
u_{2}=[l k+i \nu(z-1)](z-1)^{p-2} c-l^{i+1} d .
$$

Now $\tilde{H} u_{1}=(y-1)^{p-i} \tilde{H} c, \tilde{H} u_{2}=(y-1)^{i+1} \tilde{H} d$. Let

$$
u_{3}=k(z-1)^{p-1} c-l^{i}(z-1) d=l^{i} u_{1} \text {. }
$$


Clearly $(z-1)^{p-1} u_{3}=0$ and

$$
(x-1)^{p-1}(z-1)^{p-2} u_{3}=-(y-1)^{i} \tilde{H} d .
$$

By an argument similar to that in Lemma 3.2, we get that the dimension of the module $L$, generated by $u_{1}$ and $u_{2}$, is at least $p^{3}-p$. Since the dimension of the kernel of $\psi$ is $p^{3}-p, L \cong \Omega\left(M_{i}\right)$.

We can form the exact sequence

$$
0 \rightarrow \Omega^{2}\left(M_{i}\right) \rightarrow F \stackrel{\theta}{\rightarrow} L \rightarrow 0
$$

where $\theta(a)=u_{1}$ and $\theta(b)=u_{2}$. It is easy to see that

$$
\theta(m(i+1,1))=l^{i+1} u_{1}-(z-1) u_{2}=0 .
$$

Also

$$
\begin{aligned}
\theta(m(i+1,2)) & =k(z-1)^{p-2} u_{1}-l^{p-i-1} u_{2} \\
& =\left[k l^{p-i}-l^{p-i} k-i \nu l^{p-i-1}(z-1)\right](z-1)^{p-2} c=0,
\end{aligned}
$$

by (2.3). Consequently $M_{i+1}$ is in the kernel of $\theta$. By Lemma $3.2, M_{i+1}$ is the kernel of $\boldsymbol{\theta}$.

To conclude the proof of Theorem 3.1 we need only the following.

LEMMA 3.7. $\Omega^{2}\left(M_{p-1}\right) \simeq M$.

Proof. We have an exact sequence

$$
0 \rightarrow \Omega\left(M_{p-1}\right) \rightarrow \stackrel{\circ}{\prime} \stackrel{\circ}{\rightarrow} M_{p-1} \rightarrow 0
$$

where $F^{\prime}=K G c \oplus K G d, \sigma(c)=m(p-1,1)$ and $\sigma(d)=m(p-1,2)$. Let $u=l c-(z-1) d$. Then $\sigma(u)=0$. Now $\tilde{H} u=(y-1) \tilde{H} c$ and

$$
(x-1)^{p-1}(z-1)^{p-2} l^{p-1} u=-(y-1)^{p-1} \tilde{H} d \neq 0 .
$$

By an argument similar to that of Lemma 3.2, we get that Dim $K G u>p^{3}-$ $p$. Therefore the kernel of $\sigma$ is $K G u \cong \Omega\left(M_{p-1}\right)$.

Define $\tau: K G \rightarrow K G u$ by $\tau(1)=u$. The kernel of $\tau$ has dimension $p$ and is isomorphic to $\Omega^{2}\left(M_{p-1}\right)$. Let $\omega=(z-1)^{p-1} l^{p-1}$. Then $\tau(\omega)=0$ and $(z-1) \omega$ $=l \omega=0$. Since $(x-1)^{p-1} \omega=(y-1)^{p-1} \tilde{H} \neq 0, K G \omega$ is the kernel of $\tau$, and $M=K G \omega$. This completes the proof of the lemma and the theorem.

It should be noted that if $\alpha \in F_{p}$ then $M(\alpha)$ is not periodic. This follows from the fact that the restriction of $M(\alpha)$ to the subgroup $J=\left\langle x^{-\alpha}, z\right\rangle$ is not a periodic module (see [2]). It remains to show that there exist periodic modules with period $2 p$ when $K=F_{p}$.

Let $f=T^{n}+\beta_{n-1} T^{n-1}+\cdots+\beta_{1} T+\beta_{0}$ be an irreducible polynomial in $K[T]$. Let $L$ be the $K G$-module of dimension $n p$ on which $x$ and $y$ are represented by the matrices 


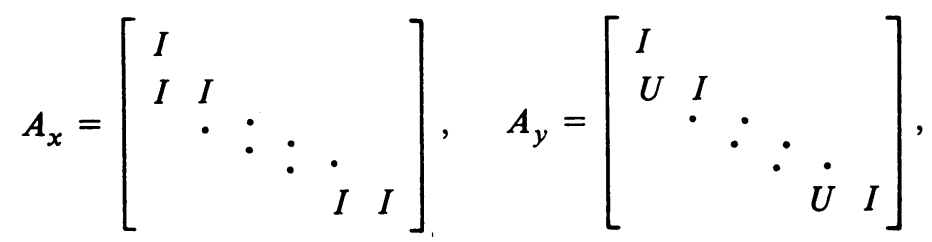

respectively, where $I$ is the $n \times n$ identity matrix, and

$$
U=\left[\begin{array}{cccccccc}
0 & 1 & & & & & \\
& 0 & 1 & & & & \\
& & \cdot & \cdot & & & \\
& & & & \cdot & \cdot & \\
& & & & & 0 & 1 \\
-\beta_{0} & -\beta_{1} & \cdot & \cdot & \cdot & & -\beta_{n-1}
\end{array}\right]
$$

is the companion matrix for $f$. Now $L$ is an indecomposable $K G$-module. If $K^{\prime}$ is an extension of $K$ which splits $f$, then

$$
K^{\prime} \otimes_{K} L \cong M\left(\alpha_{1}\right) \oplus \cdots \oplus M\left(\alpha_{n}\right)
$$

where $\alpha_{1}, \ldots, \alpha_{n}$ are the roots of $f$ in $K^{\prime}$. If therefore $n>1$, then $L$ is periodic with period $2 p$ since, by the Noether-Deuring Theorem (see [3, 29.7]),

$$
K^{\prime} \otimes \Omega^{2 p}(L) \cong \Omega^{2 p}\left(K^{\prime} \otimes L\right) \cong K^{\prime} \otimes L
$$

implies that $\Omega^{2 p}(L) \approx L$.

The reader is invited to check that $L$ is periodic with period $2 p$ when the matrix $U$ is replaced by

$$
U^{\prime}=\left[\begin{array}{llllll}
\alpha & & & & \\
1 & \alpha & & & \\
& \bullet & \ddots & & \\
& & & 1 & \alpha
\end{array}\right]
$$

for $\alpha \in K, \alpha \notin F_{p}$. Combining this with the fact that $M(\alpha) \simeq M(\beta)$ if and only if $\alpha=\beta$, we get the following.

THEOREM 3.8. Let $p$ be an odd prime and let $K$ be a field of characteristic $p$. If $G$ is the nonabelian group of order $p^{3}$ and exponent $p$, then there exist periodic $K G$-modules which have period $2 p$. Moreover there exist an infinite number of isomorphism classes of such modules and there exist such modules with arbitrarily large dimension. 


\section{REFERENCES}

1. J. L. Alperin, Perodicity in groups, Illinois J. Math. 21 (1977), 776-783.

2. J. F. Carlson, The dimensions of periodic modules over modular group algebras, Illinois J. Math. (to appear).

3. C. W. Curtis and I. Reiner, Representation theory of finite groups and associative algebras, Interscience, New York, 1966.

4. D. Eisenbud, Homological algebra on a complete intersection, with an application to group representations (to appear).

5. A. Heller, Indecomposable representations and the loop-space operation, Proc. Amer. Math. Soc. 12 (1961), 460-463.

Dipartmint of Mathematics, University of Gborgia, Athens, Ggorgin 30602 